\title{
Is there a relationship between hospital care quality assessemnt and the patient's place of residence?
}

\begin{abstract}
Introduction. Measuring the patient satisfaction with the hospital stay, as well as the knowledge of their requirements are very important in the management of health institutions. A good example of the recognition of patients' expectations is studying the level of satisfaction with specially prepared questionnaires.

Aim. The aim of the study was to investigate the relationship between the patients' place of residence and their satisfaction with the medical care they received when treated in local hospitals

Material and methods. Patient satisfaction survey was carried out in the Independent Public Health Care Centre in Kraśnik in two subsequent years: at the turn of 2011/2012 and in February 2013. The survey consisted of patients hospitalized in the surgical wards of the hospital. A testing tool for this survey was a self-designed questionnaire entitles "Patient satisfaction survey" specifically developed by nursing managers and approved by the management of the hospital.

Results. Patients of the District Hospital in Kraśnik gave their complex assessment of satisfaction with the benefits provided by the health care facility, taking into account not only the quality of strictly medical services, but the entire infrastructure of the hospital, including ancillary services. The obtained results gave a positive assessment of the analyzed branches. No statistically significant differences between the assessments of rural and urban inhabitants were found. The results allow knowledge of the strengths and weaknesses of medical care in the wards.

Conclusions. Residents of rural areas, who are slightly better than those who live in cities, assess the work of local hospital surgical wards. You can clearly see it by assessing the quality of nurses' and doctors' work and the assessment of nutrition and the appearance of patient rooms. Systematic research, measurement and evaluation of patient satisfaction with hospital care can be the basis for the improvement and ensure an appropriate level of quality.
\end{abstract}

Keywords: patient satisfaction, health care, quality of care, Kraśnik county, municipality, rural municipality.

DOI: $10.1515 /$ pjph-2015-0028

\section{INTRODUCTION}

The demand for patient satisfaction surveys is due to the fact that health care management should be done according to the rules of market economy, strategic management and health services marketing, as well as the increasing role of patients in caring for their own health.

Patients have become a central part of the health care system. They are not only health care customers, becoming active participants of the health care process instead [1]. Patient satisfaction surveys are an indispensable part of assessing the quality of health care services. This means, until the patient's opinion is elicited, such surveys cannot be treated as complete. Patient satisfaction is a key source of information about the quality of health care and there is an overall consent for including patients in the assessment process. It is the patients' basic right to have their say about the quality of service they receive. It needs emphasizing, though, that most patients provide either positive or mild opinions. Overall, patients are not too eager to provide critical opinions, even if the surveys are anonymous or they are conducted after the patient left the medical facility. In addition, there is some 'halo effect' whereby the patients' impressions of a given doctor or nurse (either totally positive or negative) influence their opinion of the health care system as a whole. The subject matter of the assessment is another thing that is important here. In this regard, patients' opinions might be way different from the opinions of health care professionals. This is due to the fact that patients, often lacking the right expertise, are unable to provide an authoritative opinion on health care.

What is patient satisfaction, then? Would it suffice to say that satisfaction is related to personal expectations, as well as one's values or experience?

Patient satisfaction is a degree to which the patient positively assesses healthcare, including their own (patients') needs or expectations. It works similarly to fulfilling the needs of a customer, regarding a particular product or service. Health care institutions should treat patients' satisfaction as a complex phenomenon, related to the services provided 
to the patient. Only such an attitude would leave the patients satisfied, since their contentment should be understood as the degree of accepting the given health care institution, needs and expectations. Furthermore, such measures make it easier to build a positive image of a given health care facility, as an institution respecting the patients' needs and listening to their expectations.

The purchasing process, namely the moment when a medical service is being provided to the patient is when two worlds collide - one of customer's expectations and the other - the service they receive. Subjective feelings combined with patient's individual needs can provide a full picture of how the patients assess the quality of care they receive in a given health care facility [2].

Patient satisfaction surveys should be used by health care managers as a method to improve the quality of medical professionals' work. Such measures could significantly facilitate the process of introducing new upgrades. The idea of measuring patients satisfaction is thus very important in managing health care and it shows to which degree does the patient appreciate the hospitals' efforts to improve the quality of work and image building $[3,4]$.

\section{AIM}

The aim of the study was to elicit patients' opinions about their satisfaction with the quality of health care they receive at their regional hospital.

\section{MATERIAL AND METHODS}

The patient satisfaction survey was conducted in Independent Public Complex of Health Care Facilities in Kraśnik, during two consecutive years: between December 2011 to January 2012 and February 2013. The subjects of the study were patients hospitalized in the institution's treatment facilities. In total, there were 105 questionnaires handed out (2012 and 2013). Some 102 patients were looked at in 2011/12 and 103 were looked at in 2013 (with survey return rates of $97.1 \%$ ). Surveys were filled out by patients of the following wards: general surgery, traumatology and the gynecology/ obstetrics ward. The participants filled out the questionnaires on their own. In case a patient was unable to make decisions on their own, the feedback was provided by either a family member or a caretaker.

In the study, the authors used a questionnaire of their own making, called "Patient Satisfaction Survey" and done with the help of manager nurses, then approved by the hospital board of directors. The questionnaire assessing the satisfaction contained 7 socio-demographic questions, as well as 32 detailed questions divided into 8 thematic blocks. These included queries about: admission to the hospital, the ward's sickrooms, diet and meals, nurse care, diagnostics, other services, visitors, doctors.

\section{RESULTS}

The group of urban residents surveyed in 2012 was chiefly composed of women - they made up $74.1 \%$. They were mainly people in their second or third decade of life, with a mean age of 45.1. They had either been referred to the hospital by their family doctor or an intensive care doctor. For $39.8 \%$ of them, it was their first stay in a hospital and the mean hospitalization time was 8.8 days. In case of rural residents, some $53 \%$ were referred to the hospital by an intensive care doctor. For $62 \%$ of them, most of which were female $(65.8 \%)$ it was the first time in a hospital. The average time of hospital stay was 6.3 days, with the mean age of 48 years. For $50.3 \%$ of patients, the stay at a treatment facility was their first time in a hospital, with a mean time of stay 5.8 days. The average age was 41.3 years and $79.2 \%$ were female.

In the questionnaire, the patients of Independent Public Complex of Health Care Facilities in Kraśnik answered queries concerning the satisfaction they took from the service they received in a given health care facility. They were also inquired about the quality of hospital infrastructure and other services. The idea behind it was to elicit patients' opinions about the service they receive from health care workers (doctors, nurses, lab assistants or physiotherapists, ) as well as receiving more information about the demand for accommodation services or know patients' expectations about meals.

The first point in the questionnaire included patients' admission to the hospital. The results were significantly better in the second year of the study which proves that health care workers have drawn some conclusions from the previous questionnaires and improved the work of the units responsible for the initial contact with the sick individual. Urban residents tended to give better grades to the issues related to hospital admission. The results are presented in Table 1.

Another thing that the questionnaires looked at were the living conditions in the hospital. This pertains to the room design, the facilities available at hand, as well as the rooms' tidiness. The findings are presented in Table 2 - the overall assessment was good, with slightly worse results in 2013, which was particularly noticeable among urban residents.

The survey also looked at the food served in the hospital. Generally speaking, patients gave positive opinions but there were numerous grades like "average", which calls for some further investigation of the subject (Table 3).

Nursing care quality was also given a closer look. During the two years the study has been performed, nurses received very good grades (Table 4).

Researchers also looked at the patients' assessment of diagnostic laboratories and the way they collect samples for work. The respondents provided good grades for that (Table 5) and there were no differences between rural and urban residents in that respect. The only things they complained about were long waiting times and the behavior of lab workers.

Further questions regarded other services provided by the hospital, like preparing the hospital discharge procedure from the hospital or ordering the essential tools to provide medical services. According to patients, both physiotherapists and medical secretaries proved excellent in this respect. The findings are presented in Table 6 .

Another part of the survey focused on visiting patients in the hospital, as well as the availability of the amenities that make it possible. The results are shown in Table 7 . 
TABLE 1. Hospital admission assessment - medical care units.

\begin{tabular}{|c|c|c|c|c|c|c|c|c|c|c|c|c|c|c|c|c|c|c|c|c|}
\hline & \multicolumn{10}{|c|}{2012} & \multicolumn{10}{|c|}{2013} \\
\hline & \multicolumn{5}{|c|}{$\begin{array}{l}\text { Patients } \\
\text { from metropolitan areas }\end{array}$} & \multicolumn{5}{|c|}{$\begin{array}{l}\text { Patients } \\
\text { from rural areas }\end{array}$} & \multicolumn{5}{|c|}{$\begin{array}{l}\text { Patients } \\
\text { from metropolitan areas }\end{array}$} & \multicolumn{5}{|c|}{$\begin{array}{c}\text { Patients } \\
\text { from rural areas }\end{array}$} \\
\hline & 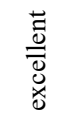 & $\begin{array}{l}\text { T. } \\
8 \\
8\end{array}$ & 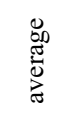 & ठே. & $\begin{array}{l}\bar{\delta} \\
\stackrel{0}{0} \\
\grave{j} \\
>\end{array}$ & 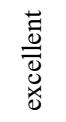 & $\begin{array}{l}\text { D̊ } \\
8 \\
8\end{array}$ & 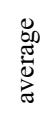 & : & 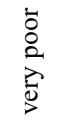 & 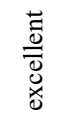 & $\begin{array}{l}\text { D } \\
8 \\
0\end{array}$ & 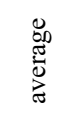 & ஜே & 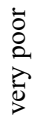 & 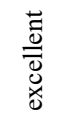 & $\begin{array}{l}\text { T. } \\
8 \\
800\end{array}$ & 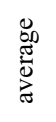 & 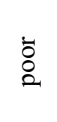 & 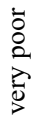 \\
\hline $\begin{array}{l}\text { Was the hospital easy } \\
\text { to access? }\end{array}$ & 51.9 & 35.7 & 10.6 & - & 2 & 36.9 & 55.7 & 7.4 & - & - & 54.2 & 37.5 & 6.8 & - & - & 43.3 & 49.8 & 7 & - & - \\
\hline $\begin{array}{l}\text { Were the administrative } \\
\text { procedures simple enough? }\end{array}$ & 37.7 & 39.2 & 19.4 & 3.7 & - & 28.6 & 57.4 & 10 & 1.7 & 2.4 & 43.8 & 41.1 & 13.5 & 1.6 & - & 37.4 & 50.8 & 7.8 & 4 & - \\
\hline $\begin{array}{l}\text { How did the medical staff } \\
\text { behave? }\end{array}$ & 42.8 & 39.8 & 13.7 & 3.7 & - & 52.4 & 34.7 & 9.6 & - & - & 47 & 36.6 & 16.5 & - & - & 52.2 & 44.6 & 2.2 & 1.1 & - \\
\hline
\end{tabular}

TABLE 2. Wards in medical care units.

\begin{tabular}{|c|c|c|c|c|c|c|c|c|c|c|c|c|c|c|c|c|c|c|c|c|}
\hline & \multicolumn{10}{|c|}{2012} & \multicolumn{10}{|c|}{2013} \\
\hline & \multicolumn{5}{|c|}{$\begin{array}{c}\text { Patients } \\
\text { from metropolitan areas }\end{array}$} & \multicolumn{5}{|c|}{$\begin{array}{c}\text { Patients } \\
\text { from rural areas }\end{array}$} & \multicolumn{5}{|c|}{$\begin{array}{c}\text { Patients } \\
\text { from metropolitan areas }\end{array}$} & \multicolumn{5}{|c|}{$\begin{array}{c}\text { Patients } \\
\text { from rural areas }\end{array}$} \\
\hline & 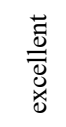 & రृ & 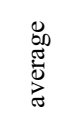 & ¿̆丶 & $\begin{array}{l}\overrightarrow{0} \\
0 \\
\stackrel{0}{0} \\
\stackrel{0}{0}\end{array}$ & 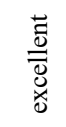 & రृ & 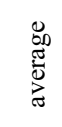 & $\ddot{\check{0}}$ & $\begin{array}{l}\overrightarrow{0} \\
0 \\
0 \\
\grave{d} \\
j\end{array}$ & 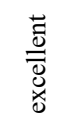 & $\overrightarrow{8}$ & 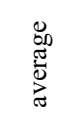 & 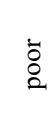 & $\begin{array}{l}\overrightarrow{0} \\
0 \\
0 \\
\stackrel{0}{0}\end{array}$ & 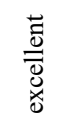 & $\overrightarrow{8}$ & 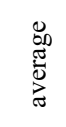 & $\check{\check{o}}$ & 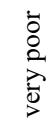 \\
\hline Ward cleanliness & 44.2 & 52.3 & 3.5 & - & - & 43.1 & 55.2 & 1.7 & - & - & 45.4 & 49.9 & 4.7 & - & - & 49.8 & 46.4 & 3.8 & - & - \\
\hline Ward temperature & 51.7 & 37.4 & 5.9 & 2 & 3 & 55 & 45 & - & - & - & 42.8 & 51.5 & 1.6 & 4.2 & - & 48.4 & 41.9 & 7 & - & 2.9 \\
\hline Noise level & 25.4 & 48.5 & 21.3 & 2.1 & 2.1 & 16.9 & 67.7 & 13.5 & 1.9 & - & 18.7 & 63 & 18.4 & - & - & 18.9 & 65.1 & 16.1 & - & - \\
\hline $\begin{array}{l}\text { The condition } \\
\text { of the ward's facilities }\end{array}$ & 25.2 & 54.5 & 18.4 & 1.8 & - & 27.1 & 60.2 & 10.9 & 1.8 & - & 20.1 & 46.3 & 29.5 & 4.2 & - & 30.3 & 56.2 & 13.6 & - & - \\
\hline $\begin{array}{l}\text { How did the cleaning } \\
\text { staff behave? }\end{array}$ & 69.4 & 20.9 & 6.7 & - & 3 & 66.7 & 30.6 & 1.7 & - & - & 42 & 54.4 & 3.6 & - & - & 61.9 & 33 & 3.1 & - & 2.1 \\
\hline
\end{tabular}

TABLE 3. Hospital food standards - patients' opinions.

\begin{tabular}{|c|c|c|c|c|c|c|c|c|c|c|c|c|c|c|c|c|c|c|c|c|}
\hline & \multicolumn{10}{|c|}{2012} & \multicolumn{10}{|c|}{2013} \\
\hline & \multicolumn{5}{|c|}{$\begin{array}{l}\text { Patients } \\
\text { from metropolitan areas }\end{array}$} & \multicolumn{5}{|c|}{$\begin{array}{c}\text { Patients } \\
\text { from rural areas }\end{array}$} & \multicolumn{5}{|c|}{$\begin{array}{c}\text { Patients } \\
\text { from metropolitan areas }\end{array}$} & \multicolumn{5}{|c|}{$\begin{array}{l}\text { Patients } \\
\text { from rural areas }\end{array}$} \\
\hline & 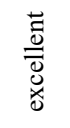 & 吾 & 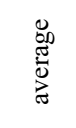 & 苂 & $\begin{array}{l}\overrightarrow{0} \\
\dot{0} \\
2 \\
\overrightarrow{0}\end{array}$ & 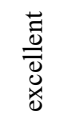 & $\begin{array}{l}\overline{0} \\
8 \\
8\end{array}$ & 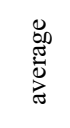 & $\ddot{\check{z}}$ & $\begin{array}{l}\dot{0} \\
\stackrel{0}{0} \\
\grave{0} \\
>\end{array}$ & 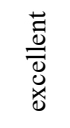 & $\begin{array}{l}\overrightarrow{0} \\
8 \\
8\end{array}$ & 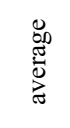 & $\ddot{\circ}$ & $\begin{array}{l}\dot{0} \\
\stackrel{0}{0} \\
\grave{0} \\
\dot{0}\end{array}$ & 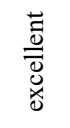 & 吾 & 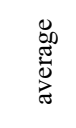 & $\ddot{\circ}$ & 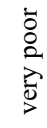 \\
\hline Available diets & 9.9 & 53.3 & 23.3 & 3.9 & 1.8 & 18.3 & 58.5 & 18.7 & 1.8 & - & 19.8 & 50.2 & 22.2 & 4.7 & 3.1 & 16.5 & 59.4 & 19.5 & 2.3 & 2.3 \\
\hline Food temperature & 5 & 56.5 & 31.1 & 5.6 & - & 14.7 & 58.9 & 24.4 & 2 & - & 24.8 & 45.7 & 18.6 & 9.4 & - & 16.1 & 56.4 & 25.3 & 1.1 & 1.1 \\
\hline Food quality & 6 & 42 & 46.4 & 3.7 & 1.9 & 12.3 & 65.3 & 20.5 & - & 1.8 & 13.6 & 40.9 & 34.6 & 7.8 & 3.1 & 12.3 & 47.8 & 36.8 & 1 & 2.1 \\
\hline Food serving time & 24.5 & 52.7 & 22.8 & - & - & 22.6 & 67.4 & 10 & - & - & 30.2 & 62 & 7.8 & - & - & 33.4 & 51.6 & 15 & - & - \\
\hline
\end{tabular}

TABLE 4. The quality of nursing care in the facility.

\begin{tabular}{|c|c|c|c|c|c|c|c|c|c|c|c|c|c|c|c|c|c|c|c|c|}
\hline & \multicolumn{10}{|c|}{2012} & \multicolumn{10}{|c|}{2013} \\
\hline & \multicolumn{5}{|c|}{$\begin{array}{c}\text { Patients } \\
\text { from metropolitan areas }\end{array}$} & \multicolumn{5}{|c|}{$\begin{array}{c}\text { Patients } \\
\text { from rural areas }\end{array}$} & \multicolumn{5}{|c|}{$\begin{array}{c}\text { Patients } \\
\text { from metropolitan areas }\end{array}$} & \multicolumn{5}{|c|}{$\begin{array}{c}\text { Patients } \\
\text { from rural areas }\end{array}$} \\
\hline & 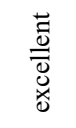 & $\begin{array}{l}-8 \\
8 \\
8\end{array}$ & 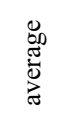 & $\ddot{\ddot{o}}$ & $\begin{array}{l}\grave{0} \\
\stackrel{\circ}{\circ} \\
\grave{0}\end{array}$ & $\begin{array}{l}\overrightarrow{0} \\
\stackrel{\overrightarrow{0}}{\overrightarrow{0}} \\
\underset{0}{0}\end{array}$ & 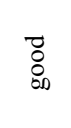 & 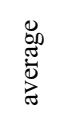 & ڤ̆ & 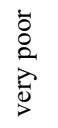 & 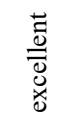 & $\begin{array}{l}\overline{8} \\
8 \\
8\end{array}$ & 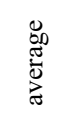 & 苂 & 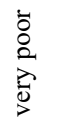 & 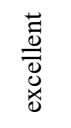 & ت & 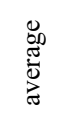 & $\ddot{\circ}$ & 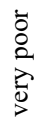 \\
\hline $\begin{array}{l}\text { How would you rate } \\
\text { the nurses' attitude? }\end{array}$ & 74.3 & 20.9 & 4.7 & - & - & 69.7 & 28.6 & 1.7 & - & - & 77.8 & 20.1 & 2.1 & - & - & 79.4 & 23.1 & - & - & - \\
\hline $\begin{array}{l}\text { Did the nurses respond } \\
\text { rapidly enough? }\end{array}$ & 70.3 & 25 & 4.7 & - & - & 59 & 39.2 & 1.8 & - & - & 65 & 32.9 & 2.1 & - & - & 71.9 & 25.9 & 2.3 & - & - \\
\hline $\begin{array}{l}\text { How did the nurses react } \\
\text { to your requests? }\end{array}$ & 55.8 & 39.1 & 5.1 & - & - & 55.6 & 42.7 & 1.8 & - & - & 68.8 & 29.2 & 2.1 & - & - & 59.3 & 40.7 & - & - & - \\
\hline $\begin{array}{l}\text { Did the nurses explain your } \\
\text { condition in a clear way? }\end{array}$ & 62.5 & 27.7 & 9.8 & - & - & 34.6 & 58.3 & 5.3 & 1.8 & - & 51.6 & 46.4 & 2.1 & - & - & 53.5 & 43.7 & 2.8 & - & - \\
\hline $\begin{array}{l}\text { Were medications } \\
\text { administered regularly? }\end{array}$ & 54.5 & 37.5 & 7.9 & - & - & 52.7 & 47.3 & - & - & - & 65.1 & 34.9 & - & - & - & 60.1 & 37.8 & 2.1 & - & - \\
\hline $\begin{array}{l}\text { Were nurses readily available } \\
\text { during the afternoon/night shift? }\end{array}$ & 54.6 & 35 & 6.7 & 1.9 & - & 51.1 & 48.9 & - & - & - & 71.9 & 24.5 & 3.6 & - & - & 64 & 33.9 & 2.1 & - & - \\
\hline
\end{tabular}


TABLE 5. The quality of diagnostics.

\begin{tabular}{|c|c|c|c|c|c|c|c|c|c|c|c|c|c|c|c|c|c|c|c|c|}
\hline & \multicolumn{10}{|c|}{2012} & \multicolumn{10}{|c|}{2013} \\
\hline & \multicolumn{5}{|c|}{$\begin{array}{c}\text { Patients } \\
\text { from metropolitan areas }\end{array}$} & \multicolumn{5}{|c|}{$\begin{array}{c}\text { Patients } \\
\text { from rural areas }\end{array}$} & \multicolumn{5}{|c|}{$\begin{array}{c}\text { Patients } \\
\text { from metropolitan areas }\end{array}$} & \multicolumn{5}{|c|}{$\begin{array}{c}\text { Patients } \\
\text { from rural areas }\end{array}$} \\
\hline & 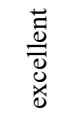 & $\begin{array}{l}7 \\
8 \\
8 \\
0\end{array}$ & 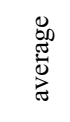 & $\dot{\vec{o}}$ & $\begin{array}{l}\overrightarrow{0} \\
\dot{0} \\
\grave{0} \\
\overrightarrow{0}\end{array}$ & $\begin{array}{l}\stackrel{\overrightarrow{0}}{\vec{J}} \\
\stackrel{0}{0} \\
\underset{0}{0}\end{array}$ & $\begin{array}{l}-8 \\
8 \\
8\end{array}$ & 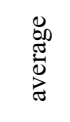 & $\ddot{\circ}$ & $\begin{array}{l}\ddot{0} \\
0 \\
\stackrel{0}{0} \\
\grave{D}\end{array}$ & 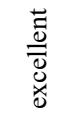 & $\begin{array}{l}-8 \\
8 \\
8\end{array}$ & 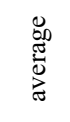 & 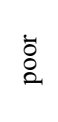 & $\begin{array}{l}\overrightarrow{0} \\
\dot{0} \\
\stackrel{0}{0} \\
\overrightarrow{0}\end{array}$ & 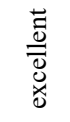 & $\begin{array}{l}\overline{0} \\
8 \\
8\end{array}$ & 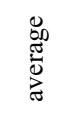 & 。̊ & 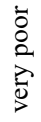 \\
\hline Blood draw & 67.2 & 29 & 3.8 & - & - & 64.6 & 35.4 & - & - & - & 82.8 & 15.1 & 2.1 & - & - & 68.5 & 31.6 & - & - & - \\
\hline What were the waiting times? & 38.9 & 44.5 & 14.5 & 2.1 & - & 34.3 & 51.7 & 12.4 & 1.7 & - & 37.5 & 46 & 14.9 & 1.6 & - & 32.9 & 57 & 5.2 & - & - \\
\hline $\begin{array}{l}\text { How did the lab employees } \\
\text { behave? }\end{array}$ & 45.9 & 46.6 & 7.5 & - & - & 46.5 & 51.9 & 1.7 & - & - & 46.9 & 47.9 & 2.1 & - & - & 42.7 & 53.4 & 3.9 & - & - \\
\hline
\end{tabular}

TABLE 6. Other services.

\begin{tabular}{|c|c|c|c|c|c|c|c|c|c|c|c|c|c|c|c|c|c|c|c|c|}
\hline & \multicolumn{10}{|c|}{2012} & \multicolumn{10}{|c|}{2013} \\
\hline & \multicolumn{5}{|c|}{$\begin{array}{c}\text { Patients } \\
\text { from metropolitan areas }\end{array}$} & \multicolumn{5}{|c|}{$\begin{array}{c}\text { Patients } \\
\text { from rural areas }\end{array}$} & \multicolumn{5}{|c|}{$\begin{array}{c}\text { Patients } \\
\text { from metropolitan areas } \\
\end{array}$} & \multicolumn{5}{|c|}{$\begin{array}{c}\text { Patients } \\
\text { from rural areas }\end{array}$} \\
\hline & 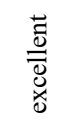 & 范 & 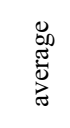 & 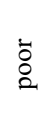 & $\begin{array}{l}\overrightarrow{0} \\
\stackrel{\circ}{\circ} \\
\stackrel{0}{0}\end{array}$ & 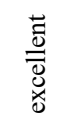 & $\begin{array}{l}\overline{8} \\
8 \\
8\end{array}$ & 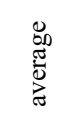 & 范 & $\begin{array}{l}\grave{0} \\
\stackrel{0}{8} \\
\stackrel{0}{0}\end{array}$ & 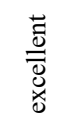 & $\begin{array}{l}\overline{8} \\
8 \\
8\end{array}$ & $\begin{array}{l}\stackrel{8}{8} \\
\stackrel{\pi}{0} \\
\stackrel{\varpi}{0}\end{array}$ & $\ddot{\circ}$ & $\begin{array}{l}\overrightarrow{0} \\
\stackrel{8}{\circ} \\
\stackrel{\overrightarrow{0}}{>}\end{array}$ & 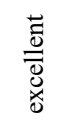 & 范 & 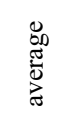 & $\begin{array}{l}\text { ŏ } \\
\text { }\end{array}$ & 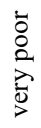 \\
\hline $\begin{array}{l}\text { How would you rate } \\
\text { the work of physiotherapists? }\end{array}$ & 32.5 & 47.9 & 23.4 & - & - & 21.3 & 61.1 & 14.8 & - & 2.8 & 28.2 & 57.7 & 14.1 & - & - & 39 & 59.5 & 1.5 & - & - \\
\hline $\begin{array}{l}\text { How would you rate the work } \\
\text { of medical secretaries? }\end{array}$ & 42.5 & 54.2 & 3.3 & - & - & 41.5 & 54.2 & 4.2 & - & - & 25 & 75.1 & - & - & - & 39.9 & 55.4 & 2.5 & - & - \\
\hline $\begin{array}{l}\text { Could you rate the work } \\
\text { of hospital administration } \\
\text { employees? }\end{array}$ & 28.9 & 61.7 & 9.4 & - & - & 24.5 & 64.5 & 11 & - & - & 39.1 & 38.9 & 22 & - & - & 42.8 & 46.8 & 10.2 & - & - \\
\hline
\end{tabular}

TABLE 7. Visiting patients in the hospital.

\begin{tabular}{|c|c|c|c|c|c|c|c|c|c|c|c|c|c|c|c|c|c|c|c|c|}
\hline & \multicolumn{10}{|c|}{2012} & \multicolumn{10}{|c|}{2013} \\
\hline & \multicolumn{5}{|c|}{$\begin{array}{c}\text { Patients } \\
\text { from metropolitan areas }\end{array}$} & \multicolumn{5}{|c|}{$\begin{array}{c}\text { Patients } \\
\text { from rural areas }\end{array}$} & \multicolumn{5}{|c|}{$\begin{array}{c}\text { Patients } \\
\text { from metropolitan areas }\end{array}$} & \multicolumn{5}{|c|}{$\begin{array}{c}\text { Patients } \\
\text { from rural areas }\end{array}$} \\
\hline & 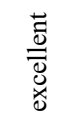 & $\begin{array}{l}7 \\
8 \\
8\end{array}$ & $\begin{array}{l}\stackrel{8}{8} \\
\stackrel{\pi}{0} \\
\stackrel{0}{\pi}\end{array}$ & 范 & 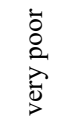 & 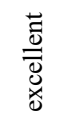 & تृ & 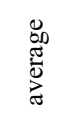 & $\ddot{\ddot{o}}$ & $\begin{array}{l}\grave{0} \\
\stackrel{0}{2} \\
\lambda_{0} \\
>\end{array}$ & 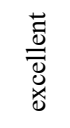 & 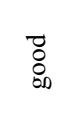 & $\begin{array}{l}\stackrel{8}{8} \\
\frac{\pi}{0} \\
\stackrel{\varpi}{\pi}\end{array}$ & : & 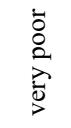 & 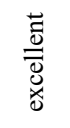 & ت & 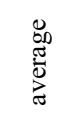 & $\ddot{\circ}$ & 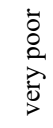 \\
\hline $\begin{array}{l}\text { Was the visiting time } \\
\text { appropriate? }\end{array}$ & 53.3 & 41 & - & 5.7 & - & 56.3 & 43.7 & - & - & - & 54.7 & 37.5 & 7.8 & - & - & 69.1 & 29.2 & 1.8 & - & - \\
\hline $\begin{array}{l}\text { How did the staff behave } \\
\text { toward the visitors? }\end{array}$ & 66.9 & 31.2 & 2 & - & - & 46 & 52.4 & 1.7 & - & - & 67.1 & 26.6 & 6.3 & - & - & 63.6 & 36.4 & - & - & - \\
\hline Shops and facilities & 3.3 & 37.1 & 13.7 & 10.4 & 32.2 & - & 55 & 15.9 & 24.3 & 7.1 & 10.4 & 20.8 & 11.7 & 42.9 & 14.3 & 6.3 & 38.3 & 33.2 & 26.8 & 6.3 \\
\hline Hospital parking & 9.7 & 25.4 & 41.1 & 11.4 & 9 & 4.3 & 47.5 & 30.1 & 14.7 & 3.3 & 9.7 & 43.1 & 34.7 & 6.3 & 6.2 & 9.9 & 33.1 & 31.3 & 19.1 & 6.6 \\
\hline
\end{tabular}

TABLE 8. Rating physicians' work.

\begin{tabular}{|c|c|c|c|c|c|c|c|c|c|c|c|c|c|c|c|c|c|c|c|c|}
\hline & \multicolumn{10}{|c|}{2012} & \multicolumn{10}{|c|}{2013} \\
\hline & \multicolumn{5}{|c|}{$\begin{array}{c}\text { Patients } \\
\text { from metropolitan areas }\end{array}$} & \multicolumn{5}{|c|}{$\begin{array}{l}\text { Patients } \\
\text { from rural areas }\end{array}$} & \multicolumn{5}{|c|}{$\begin{array}{c}\text { Patients } \\
\text { from metropolitan areas }\end{array}$} & \multicolumn{5}{|c|}{$\begin{array}{c}\text { Patients } \\
\text { from rural areas }\end{array}$} \\
\hline & $\frac{\overrightarrow{0}}{\bar{\Xi}}$ & $\begin{array}{l}8 \\
8 \\
8\end{array}$ & 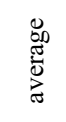 & ठே. & 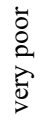 & 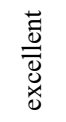 & $\begin{array}{l}\text { Z } \\
8 \\
8\end{array}$ & 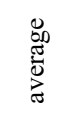 & ¿̈ & 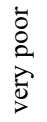 & $\frac{\overrightarrow{0}}{\stackrel{\overrightarrow{0}}{\bar{d}}}$ & Z & 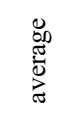 & ‡̄ & $\begin{array}{l}5 \\
0 \\
0 \\
0 \\
\grave{0} \\
0\end{array}$ & 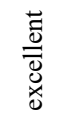 & Z & 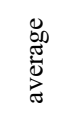 & ¿̈ & 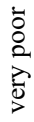 \\
\hline $\begin{array}{l}\text { Did physicians spend enough } \\
\text { time with you? }\end{array}$ & 13.7 & 63 & 17.7 & 5.6 & - & 22.7 & 63 & 12.7 & 1.7 & - & 42.8 & 43.7 & 10.4 & 3.1 & - & 32.6 & 55.5 & 11.9 & - & - \\
\hline Physicians' behavior & 49.2 & 37.5 & 11.4 & 1.8 & - & 45.3 & 36.2 & 16.9 & 1.7 & - & 38.3 & 49.8 & 12 & - & - & 47.3 & 54.5 & 5.4 & - & - \\
\hline $\begin{array}{l}\text { Did physicians explain your } \\
\text { condition in a clear way? }\end{array}$ & 40 & 40.9 & 17.2 & 1.9 & - & 30.7 & 55.8 & 13.5 & - & - & 30.9 & 56.3 & 12.8 & - & - & 43.5 & 44.6 & 11.9 & - & - \\
\hline $\begin{array}{l}\text { Were physicians available } \\
\text { during the afternoon/night } \\
\text { shift? }\end{array}$ & 37.8 & 42.6 & 15.7 & 3.9 & - & 20.5 & 61.3 & 18.7 & - & - & 28.6 & 59.5 & 12 & - & - & 35.6 & 55.5 & 8.9 & - & - \\
\hline
\end{tabular}


TABLE 9. Patient satisfaction survey conducted in the hospital in Kraśnik (treatment facilities).

\begin{tabular}{|c|c|c|c|c|c|c|c|c|c|c|c|c|c|c|c|c|c|c|c|c|}
\hline & \multicolumn{10}{|c|}{2012} & \multicolumn{10}{|c|}{2013} \\
\hline & \multicolumn{5}{|c|}{$\begin{array}{c}\text { Patients } \\
\text { from metropolitan areas }\end{array}$} & \multicolumn{5}{|c|}{$\begin{array}{c}\text { Patients } \\
\text { from rural areas }\end{array}$} & \multicolumn{5}{|c|}{$\begin{array}{l}\text { Patients } \\
\text { from metropolitan areas }\end{array}$} & \multicolumn{5}{|c|}{$\begin{array}{c}\text { Patients } \\
\text { from rural areas }\end{array}$} \\
\hline & $\frac{\overrightarrow{0}}{\stackrel{\vec{J}}{\vec{d}}}$ & $\begin{array}{l}\text { Tे } \\
8 \\
8\end{array}$ & $\begin{array}{l}0 \\
\infty \\
\mathbb{\pi} \\
\mathbb{J} \\
\mathbb{J}\end{array}$ & $\ddot{\circ}$ & $\begin{array}{l}\ddot{0} \\
0 \\
\grave{0} \\
\overrightarrow{0}\end{array}$ & $\frac{\overrightarrow{0}}{\stackrel{\vec{J}}{\vec{d}}}$ & $\begin{array}{l}\text { Tे } \\
8 \\
0\end{array}$ & 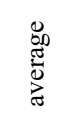 & ந் & 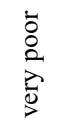 & $\frac{\overrightarrow{0}}{\stackrel{\vec{J}}{\vec{d}}}$ & $\begin{array}{l}\text { Dे } \\
8 \\
8\end{array}$ & 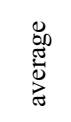 & $\ddot{\check{o}}$ & 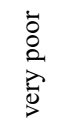 & $\frac{\overrightarrow{0}}{\vec{\Xi}}$ & $\begin{array}{l}\text { Tे } \\
8 \\
80\end{array}$ & $\begin{array}{l}0 \\
\stackrel{0}{\pi} \\
\bar{d} \\
\vec{d}\end{array}$ & 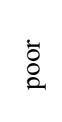 & 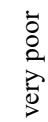 \\
\hline $\begin{array}{l}\text { Department of General } \\
\text { and Endoscopic Surgery }\end{array}$ & 31.7 & 47.1 & 15.2 & 4.4 & 1.6 & 25.8 & 58.1 & 13.8 & 1.6 & 0.7 & 30.6 & 45.8 & 16.4 & 5.2 & 2 & 35 & 51.4 & 10.6 & 1.7 & 1.3 \\
\hline $\begin{array}{l}\text { Department of Trauma } \\
\text { and Orthopedic Surgery }\end{array}$ & 46.1 & 38.7 & 12.5 & 1.5 & 1.2 & 38.3 & 53.5 & 6.6 & 1.4 & 0.2 & 64.8 & 25.4 & 9.8 & - & - & 62 & 26.5 & 10.3 & 0.7 & 0.5 \\
\hline $\begin{array}{l}\text { Department of Gynecology } \\
\text { and Obstetrics }\end{array}$ & 44 & 37.6 & 13.8 & 2.2 & 2.4 & 46.3 & 44 & 7 & 2 & 0.7 & 36.4 & 50.9 & 9.1 & 2.9 & 0.7 & 38.2 & 51.8 & 7.3 & 2.2 & 0.5 \\
\hline
\end{tabular}

The last part of the questionnaire was devoted to doctors' work (Table 8). Overall, patients provided good grades, both in the first and second year the survey was conducted. It needs emphasizing that rural residents assessed treatment facilities much better. More details are presented in Table 9 .

\section{DISCUSSION}

Patient satisfaction is a degree to which the patient positively assesses healthcare. It is an emotional and subjective opinion, hugely influenced by patient's values and expectations, as well as earlier hospital experiences [5]. Patients' opinion can be a major source of information about the quality of service provided by the health care workers and it might provide a basis for implementing a quality improvement program in a hospital [6].

A quick analysis of the bibliography shows that there is very little research looking at issues of patients' satisfaction and, at the same time, considering their place of residence. Hence, the difficulties in analyzing the needs and expectations of both rural and urban residents. Previous research made no distinction between the patients from the rural and urban areas.

In the past, there was a study on patients' satisfaction, conducted on cardiologic wards in Cracow. The survey looked at the following things: accommodation, amenities, tidiness of the rooms, meals, safety and living conditions. Some 91.7 of patients think that the way the furniture is organized (the bed, shelves, chairs, table, TV set) tidiness and design were fine [7].

Research done in hospitals located in Warmian-Masurian Voivodeship shows that over a half of the patients (53.2\%) think that nurses always understand their needs. At the same time, the patients claiming that nurses rarely understood their needs $(10 \%)$ were mostly rural residents $(21.4 \%)$. According to $68.8 \%$ of the respondents, usually female $(72.1 \%)$, nurses always cared for the good of the patient, while according to $67.9 \%$ of rural residents, nurses rarely paid any attention to it. Around $64.9 \%$ of the respondents claimed that the nurses paid enough attention to every patient. Such opinions were mostly voiced by urban residents (69.4\%) [8].

A study by PBS looks at patient satisfaction during both in-patient and outpatient hospital care. The findings show that the patients, when asked about the most recent hospitalization, had very high opinions about the way they had been treated by doctors and other medical staff ( $77 \%$ were satisfied, 78\% definitely satisfied.) General quality of hospital care received a positive mark - supported by $70 \%$ of the respondents [9].

Patients hospitalized in a hospital located in Tomaszow Mazowiecki provided positive opinions about the medical care employees (meaning doctors and nurses) working there. Over $50 \%$ of the respondents claimed that the staff work very well, $39.2 \%$ gave a "good" mark, whilst only $1.9 \%$ of respondents deemed it negative. The results show that $89.2 \%$ of the respondents have positive opinions about the kindness of the staff of the laryngological ward. The individuals surveyed assessed doctors' communication skills in the following way: $44.1 \%$ assessed it as "very good", 42.1 "good" and $2.1 \%$ deemed it negative. One of the most important things that patients looked at was doctor's care, with providing information about the kind of planned operations. Doing it that way drives the patient's stress levels down, at the same time boosting their well-being [10,11].

The SERVQUAL research by Rosiek and Leksowski looked at 6 medical care facilities from Kuyavian-Pomeranian Voivodeship. They were divided into two groups, according to the system provided by National Health Fund. The first group comprised 3 hospitals (with 400 beds in each), whilst the other composed of 3 hospitals with less than 400 beds. There were 30 patients from each hospital which gives a total of 180 respondents. Participants received questionnaires which show that one's place of residence can hugely affect their expectations and assessment of doctors' work. For instance, residents of rural areas had much higher expectations when it comes to material things. On average, they also gave more favorable grades for the quality of care they received. Patients treated in the surgical ward also expected good accommodation conditions, the design and amenities of hospital rooms, as well as the overall comfort or esthetics [12].

\section{CONCLUSIONS}

1. Rural residents tend to assess treatment facilities in their local hospital slightly better than urban residents.

2. This tendency is particularly visible in questionnaires assessing the quality of nurses' or doctors' work, as well as meals or the design of sickrooms.

3. Urban residents gave more favorable opinions about hospital admissions than rural residents. 
4. The expertise of medical staff was a huge plus for the care they received

5. Weak points included: lack of information exchange between the staff and patients, accommodation standards and meals.

6. Regular checking, measuring and assessment of patients satisfaction can become a basis for improvements and ensuring the right quality.

\section{REFERENCES}

1. Minister of Supply and Services Canada. Strategies for Population Health Investing in the Health of Canadians. Ottawa: Publications, Health Canada; 1994.

2. Hupert Z. Jakość w opiece medycznej: koncepcja pomiaru. Lublin: Instytut Medycyny Wsi; 2003.

3. Marcinowicz L, Grębowski R. Satysfakcja pacjentów w świetle polskich badań empirycznych - próba wyjaśnienia zagadki wysokiego poziomu zadowolenia z opieki. Pol Merk Lek. 2005;17:663-6.

4. Małecka B, Marcinkowski TJ. Satysfakcja pacjenta czynnikiem kształtującym współczesny rynek usług medycznych. Probl Hig Epidemmiol. 2007;88:17-9.

5. Kulczycka K. Przedmiot i kryteria jakości świadczeń zdrowotnych z punktu widzenia pacjenta. In: A. Ksykiewicz-Dorota (ed). Podstawy organizacji pracy pielęgniarskiej. Lublin: Wydawnictwo Czelej; 2004. p. $167-71$
6. Szczerbińska K. Jakość opieki zdrowotnej. In: A. Czupryna, S. Poździoch, A. Ryś, C. Włodarczyk (ed). Kraków: Vesalius; 2001. p. 141-84.

7. Gaweł G, Twarduś K, Kin-Dąbrowska J, Pyć L. Jakość opieki pielęgniarskiej na oddziale kardiologicznym. Probl Pielęg. 2008;16:33942.

8. Majchrzak-Kłokocka E, Taraszkiewicz H, Danielewicz M, Abramczyk A. Satysfakcja pacjentów hospitalizowanych $\mathrm{z}$ opieki realizowanej przez pielęgniarkę. PiZ. 2012;13(11):201-14.

9. Satysfakcja pacjentów z funkcjonowania publicznej Służby Zdrowia. Warszawa: Raport. PBS; 2005.

10. Fronczak A, Kowalska A, Sułkowska J, et al. Ocena jakości świadczeń medycznych udzielanych pacjentom oddziału laryngologicznego Szpitala w Tomaszowie Mazowieckim przez personel medyczny. PiZ. 2012;9:25-31

11. Fronczak A, Kowalska A, Sułkowska J, Walecka, et al. Wpływ opieki pielęgniarskiej na jakość usług medycznych świadczonych w Oddziale Laryngologicznym Szpitala w Tomaszowie Mazowieckim. PiZ. 2012;9:33-9.

12. Rosiek A, Leksowski K. Infrastruktura szpital, a satysfakcja pacjenta z leczenia szpitalnego. Analiza czynników wpływających na satysfakcję z leczenia szpitalnego u chorych po cholecystektomii laparoskopowej. Toruń: Wydział Nauk o Zdrowiu Collegium Medium w Bydgoszczy UMK w Toruniu; 2012. p. 5-15.

\section{Corresponding author}

Dr Marek Kos

13 Chopina Str., 23-200 Kraśnik

tel. 695-330-700

E-mail: marekkos@op.pl 